BULLETIN OF THE

AMERICAN MATHEMATICAL SOCIETY

Volume 79, Number 6, November 1973

\title{
THE ALGEBRA OF BOUNDED ANALYTIC FUNCTIONS
}

\author{
BY T. W. GAMELIN
}

Introduction. This survey features the algebra $H^{\infty}(D)$ of bounded analytic functions on a domain $D$. The central theme will be the interplay between classical function theory and functional analysis.

Typically one learns about the spectrum of $H^{\infty}(D)$, or some other abstract entity, by rephrasing the problem in terms of function theory and treating the reformulated problem with techniques of classical hard analysis. Problems of this sort have led to difficult and fecund work in hard analysis, the most notable example being Carleson's solution of the corona problem [6].

In the reverse direction, uniform-algebra techniques are beginning to yield modest but new results in function theory. An example $[\mathbf{1 8}]$ is the strong version of the Iversen-Tsuji theorem, valid for polydomains, which can be obtained from a statement concerning the Šilov boundary of $H^{\infty}(D)$. Moreover, the abstract concepts, such as "distinguished homomorphism," have served to enhance our understanding of the classical function theory.

The Banach-algebra approach to $H^{\infty}(D)$ really got under way during the Conference in Analytic Function Theory at Princeton University in 1957. At that time, virtually nothing was known about the maximal ideal space $\mathscr{M}(D)$ of $H^{\infty}(D)$. The problems brought to this conference included the following questions. Do there exist interpolating sequences in the open unit disc $\Delta$ for $H^{\infty}(\Delta)$, that is, are there sequences $S$ in $\Delta$ such that $\left.H^{\infty}(\Delta)\right|_{S}=l^{\infty}(S)$ ? Does $\mathscr{M}(\Delta) \backslash \Delta$ coincide with the Silov boundary of $H^{\infty}(\Delta)$ ? Is $\Delta$ dense in $\mathscr{M}(\Delta)$ ? This latter problem was christened the "corona problem," the "corona" being the part of $\mathscr{M}(\Delta)$ which is not adherent to $\Delta$.

This area has seen extensive development since the 1957 conference, and our aim is to give an indication of where we stand in year 15 . However, this is not to be a complete survey, and the selection of topics and of bibliographical references will be seen to be quite prejudiced. Many

An expanded version of an invited address delivered to the 702nd meeting of the Society at Stanford, on April 14, 1973; received by the editors, May 11, 1973. Research partially supported by NSF Grant GP-33693X.

AMS (MOS) subject classifications (1970). Primary 46J15, 30A98; Secondary 46J20, 30A40, 30A72, 30A82.

Key words and phrases. Bounded analytic functions, maximal ideal space, Silov boundary, corona problem, distinguished homomorphism, Iversen-Tsuji theorem, dual extremal problems, Ahlfors function. 
interesting areas of research, such as the recent work on subalgebras of $H^{\infty}(\Delta)$ due to D. Sarason and others, and work on pointwise bounded approximation, are touched on only briefly, if at all.

Most topics in this survey are covered in more detail in the expository lecture notes in the La Plata series [20]. For related articles of an expository or semiexpository nature, see [10], [21], [38] and [42].

Notation AND CONVENTIONs. By $D$ we will denote a fixed bounded open subset of the complex plane $C$. Starting with $\S 8, D$ will be connected. The open disc in $C$ with center $z$ and radius $r$ is denoted by $\Delta(z ; r)$. The open unit disc is denoted simply by $\Delta$. The sup norm of a function $f$ over a set $E$ is denoted by $\|f\|_{E}$ :

$$
\|f\|_{E}=\sup \{|f(z)|: z \in E\} .
$$

1. The spectrum of $H^{\infty}(D)$. The algebra $H^{\infty}(D)$ is a commutative Banach algebra, with the norm $\|\cdot\|_{D}$ of uniform convergence on $D$. The spectrum of $H^{\infty}(D)$, denoted by $\mathscr{M}(D)$, is the set of nonzero complexvalued homomorphisms of $H^{\infty}(D)$. Endowed with the weak topology determined by $H^{\infty}(D), \mathscr{M}(D)$ becomes a compact Hausdorff space.

Each function $f \in H^{\infty}(D)$ determines a continuous function $\hat{f}$ on $\mathscr{M}(D)$, defined by $\hat{f}(\varphi)=\varphi(f), \varphi \in \mathscr{M}(D)$. The correspondence $f \rightarrow \hat{f}$ is the Gelfand transform, and it maps $H^{\infty}(D)$ isometrically onto a closed subalgebra of $C(\mathscr{M}(D))$.

The domain $D$ can be identified with an open subset of $\mathscr{M}(D)$, by identifying each point $z \in D$ with the homomorphism $\varphi_{z}$ which evaluates $f \in H^{\infty}(D)$ at $z$. Since $\hat{f}\left(\varphi_{z}\right)=f(z)$ for $z \in D$, we can regard $\hat{f}$ as a continuous extension of $f$ from $D$ to $\mathscr{M}(D)$. We will drop the "hat", and regard the functions in $H^{\infty}(D)$ as continuous functions on $\mathscr{M}(D)$.

The extension of the coordinate function to $\mathscr{M}(D)$ is denoted by $Z$. It is easy to see that $Z$ maps $\mathscr{M}(D)$ onto the closure $\bar{D}$ of $D$, so that $\mathscr{M}(D)$ is the disjoint union of the fibers

$$
\mathscr{M}_{\zeta}(D)=Z^{-1}(\{\zeta\}), \quad \zeta \in \bar{D} .
$$

If $\zeta \in D$, then $\mathscr{M}_{\zeta}(D)$ consists of only the one point $\left\{\varphi_{\zeta}\right\}$. We are interested in the fibers $\mathscr{M}_{\zeta}(D)$ for $\zeta \in \partial D$, and we are particularly interested in relating the behavior on $\mathscr{M}_{\zeta}(D)$ of $f \in H^{\infty}(D)$ to the cluster behavior of $f$ at $\zeta$.

A point $\zeta \in \partial D$ is an inessential boundary point of $D$ if every $f \in H^{\infty}(D)$ extends to be analytic at $\zeta$. In this case, the fiber $\mathscr{M}_{\zeta}(D)$ again consists of only the homomorphism $\varphi_{\zeta}$ which evaluates the analytic extension of $f$ at $\zeta$. The remaining boundary points are called essential boundary points, and these form a closed subset of $\partial D$. If $\zeta$ is an essential boundary point, then $\mathscr{M}_{\zeta}(D)$ is quite large. 
The corona problem, in the current context, asks the following.

Corona problem. Is $D$ dense in $\mathscr{M}(D)$ ?

The corona problem remains unsolved in this generality. While this is a very natural problem, it is only fair to say that the proof that $D$ is dense in $\mathscr{M}(D)$ in any given case is certain to be more interesting and far-reaching than the fact itself.

As mentioned earlier, the corona problem was solved affirmatively by L. Carleson [6] in the case of the open unit disc. In [30], L. Hörmander showed how the corona theorem could be reduced to the problem of solving a certain system of partial differential equations, with bounds on the solutions. This idea and other simplifications are incorporated into the proof of the corona theorem given in [8].

Carleson's theorem has been extended to various classes of planar domains, and all of the extensions depend on Carleson's original theorem. E. L. Stout [44] answered the problem affirmatively for finitely connected domains, and M. Behrens [3] found a class of infinitely connected domains for which the corona problem has an affirmative solution. Behrens' construction can be used to show that either the corona problem has an affirmative solution for all open subsets of $\boldsymbol{C}$, or else it has a negative solution for certain relatively simple domains, obtained from the open unit disc $\Delta$ by excising a sequence of closed disjoint subdiscs which converge to some prescribed point of $\partial \Delta$ (or of $\Delta$ ). Recently J. Garnett (unpublished) has applied Vitushkin's techniques to widen the class of domains for which there is an affirmative solution to the corona problem.

In [15] it is shown that the fiber $\mathscr{M}_{\zeta}(D)$ depends only on the behavior of $D$ near $\zeta \in \partial D$, so that the corona problem is a "local" problem. A consequence of this localization principle is that

$$
\mathrm{Cl}(f, \zeta)=f\left(\mathscr{M}_{\zeta}(D)\right), \quad \zeta \in \partial D, f \in H^{\infty}(D) .
$$

Here $\operatorname{Cl}(f, \zeta)$ is the cluster set of $f$ at $\zeta$, consisting of all complex numbers $w$ for which there is a sequence $\left\{z_{n}\right\}$ in $D$ satisfying $z_{n} \rightarrow \zeta$ and $f\left(z_{n}\right) \rightarrow w$. Since $\mathrm{Cl}(f, \zeta)$ can be reinterpreted as the range of $f$ on the adherence of $D$ in $\mathscr{M}_{\zeta}(D)$, the cluster value theorem (1) would be a trivial consequence of the density of $D$ in $\mathscr{M}(D)$.

The corona problem, when reinterpreted as a problem in function theory, asks whether, given $f_{1}, \ldots, f_{n} \in H^{\infty}(D)$ such that

$$
\left|f_{1}\right|+\cdots+\left|f_{n}\right| \geqq \delta>0 \text { on } D,
$$

there exist $g_{1}, \ldots, g_{n} \in H^{\infty}(D)$ such that

$$
f_{1} g_{1}+\cdots+f_{n} g_{n}=1 \text {. }
$$

One can ask whether $D$ has the following stronger property. 
There exist constants $c(n, \delta, D), n \geqq 1, \delta>0$, such that if $f_{1}, \ldots, f_{n} \in H^{\infty}(D)$ satisfy $(2)$, and $\left|f_{j}\right| \leqq 1$, $1 \leqq j \leqq n$, then there exist $g_{1}, \ldots, g_{n} \in H^{\infty}(D)$ satisfying (3) and $\left|g_{j}\right| \leqq c(n, \delta, D), 1 \leqq j \leqq n$.

Carleson actually showed that the open unit disc has property $\left({ }^{*}\right)$, and he gave estimates for the constants appearing there. It is shown in [15] that finitely connected domains have property $(*)$, and that the constants $c(n, \delta, D)$ can be chosen to depend only on $n, \delta$, and the number of boundary components of $D$. If the constants associated with finitely connected domains can be selected to be independent of the connectivity of the domain, then the corona theorem is valid for all domains. Conversely, if the corona theorem is valid for all domains, then all $D$ have property $(*)$, and the constants appearing there can be chosen to be independent of $D$.

2. More general domains. Suppose that $R$ is a complex analytic variety with the property that $H^{\infty}(R)$ separates the points of $R$. The spectrum $\mathscr{M}(R)$ of $H^{\infty}(R)$ is defined as before, and the natural map $R \rightarrow \mathscr{M}(R)$ is a continuous injection. It is not known, however, whether this map embeds $R$ homeomorphically as an open subset of $\mathscr{M}(R)$. The answer is affirmative if $R$ happens to be an open subset of the extended complex plane, or of a compact Riemann surface $S$. Then there is a natural fibration of $\mathscr{M}(R)$ over the closure of $R$ in $S$. The fiber over $\zeta \in S$ depends only on the behavior of $R$ near $\zeta$, so that problems involving $\mathscr{M}(R)$ can be quickly reduced to problems involving bounded open subsets of $C$.

It is easy to find examples of domains $R$ in $C^{n}$ for which $R$ is not dense in $\mathscr{M}(R)$ - any bounded domain which is not holomorphically convex will serve. E. Bishop (cf. [15]) constructed a one-dimensional analytic variety $R$ which is not dense in $\mathscr{M}(R)$, and $\mathrm{B}$. Cole (unpublished) has constructed a Riemann surface with the same property. Cole's example can be modified to provide an example of a bounded domain of holomorphy in $\boldsymbol{C}^{3}$ for which the corona theorem fails. Meanwhile the corona problem is not yet resolved for the unit polydisc or the unit ball in $\boldsymbol{C}^{2}$.

For related work on other algebras of analytic functions in one and several complex variables, see [9], [30], [32] and [33].

3. Analytic structure in $\mathscr{M}(D)$. A subset $S$ of $\mathscr{M}(D)$ is an analytic disc if there is a continuous injection $\Phi$ of $\Delta$ onto $S$, such that $f \circ \Phi$ is analytic on $\Delta$ for every $f \in H^{\infty}(D)$. Analytic discs in the fibers $\mathscr{M}_{\zeta}(\Delta), \zeta \in \partial \Delta$, were discovered by I. J. Schark (cf. [28, p. 166]). K. Hoffman's extension (to logmodular algebras) of the Wermer embedding theorem (for Dirichlet algebras) shows that any "maximal analytic structure" in $\mathscr{M}(\Delta)$ is an analytic disc. These analytic discs are described by Hoffman [29], who 
proves, among other things, that $\varphi \in \mathscr{M}(\Delta)$ lies in an analytic disc if and only if $\varphi$ belongs to the closure of an interpolating sequence in $\Delta$.

In [3], Behrens gives a reasonable description of the fibers $\mathscr{M}_{\zeta}(D)$ associated with certain infinitely connected domains $D$. He finds that these fibers are eye-boggling objects, and that there is a wide range of possibilities for their maximal analytic structures. Many problems arise in this connection. Does every fiber over essential boundary points of $D$ contain an analytic disc? Does every distinguished homomorphism (to be defined) lie on an analytic disc? Is every analytic structure in $\mathscr{M}_{\zeta}(D)$ one-dimensional?

Schark's idea serves also to embed bidiscs in the fibers over the distinguished torus of $\mathscr{M}(\Delta \times \Delta)$. One can ask whether every maximal analytic structure in $\mathscr{M}(\Delta \times \Delta)$ is a disc or a bidisc.

4. Weak topologies for $H^{\infty}(D)$. Let $\sigma_{D}$ denote the restriction of the area measure $\sigma=d x d y$ to $D$. By the weak-star topology of $H^{\infty}(D)$ we mean always the weak-star topology which $H^{\infty}(D)$ inherits from $L^{\infty}\left(\sigma_{D}\right)$. A bounded net in $H^{\infty}(D)$ is weak-star convergent if and only if it is uniformly convergent on each compact subset of $D$. The Kreǐn-Schmulian theorem shows that $H^{\infty}(D)$ is a weak-star closed subalgebra of $L^{\infty}\left(\sigma_{D}\right)$. Consequently $H^{\infty}(D)$ can be realized as the conjugate space of the quotient Banach space $L^{1}\left(\sigma_{D}\right) /\left[L^{1}\left(\sigma_{D}\right) \cap H^{\infty}(D)^{\perp}\right]$, and the weak-star topology it inherits from $L^{\infty}\left(\sigma_{D}\right)$ coincides with the weak-star topology it enjoys as a conjugate space.

There are a host of "weak" topologies of $H^{\infty}(D)$, which coincide with the weak-star topology on bounded subsets of $H^{\infty}(D)$. These are discussed in the survey article of L. A. Rubel [38]. One such topology, for instance, is the strict topology introduced by R. C. Buck [5], in which a net $\left\{f_{\alpha}\right\}$ converges to $f$ if and only if $h f_{\alpha}$ converges uniformly to $h f$ for every continuous function $h$ on $\bar{D}$ which vanishes on $\partial D$. L. A. Rubel and J. Ryff [39] have shown that this topology coincides with the bounded weak-star topology of $H^{\infty}(D)$. One open problem along these lines is to give a tractable description of the Mackey topology of $H^{\infty}(D)$.

5. Distinguished homomorphisms. The homomorphisms $\varphi \in \mathscr{M}(D)$ which are weak-star continuous are called the distinguished homomorphisms of $H^{\infty}(D)$. Every $z \in D$ is a distinguished homomorphism. It is easy to construct domains $D$ for which there are distinguished homomorphisms not lying in $D$ (see [41], [47]). It is proved in [22] that there is at most one distinguished homomorphism in any given fiber $\mathscr{M}_{\zeta}(D)$; call it $\varphi_{\zeta}$ if it exists. Moreover, the following are equivalent, for $\zeta \in \partial D$ :

(i) There is a distinguished homomorphism $\varphi_{\zeta} \in \mathscr{M}_{\zeta}(D)$.

(ii) $\mathscr{M}_{\zeta}(D)$ is not a peak set for $H^{\infty}(D)$. 


$$
\lim _{\varepsilon \rightarrow 0} \frac{1}{\pi \varepsilon^{2}} \iint_{D \cap \Delta(\zeta ; \varepsilon)} f(z) d x d y
$$

exists for all $f \in H^{\infty}(D)$ and is nonzero for some $f \in H^{\infty}(D)$.

(iv) If $0<a<1$, and $E_{n}$ is the annulus $\left\{a^{n+1} \leqq|z-\zeta| \leqq a^{n}\right\}$, then

$$
\sum_{n=1}^{\infty} a^{-n_{\gamma}}\left(E_{n} \backslash D\right)<\infty
$$

Here $\gamma(E)$ is the analytic capacity of a set $E$, defined to be the supremum of $\left|f^{\prime}(\infty)\right|$, over all functions $f$ which are analytic off a compact subset of $E$ and which satisfy $|f| \leqq 1$. The set of points $\zeta \in \partial D$ at which there are distinguished homomorphisms is an $F_{\sigma}$-set which, according to a result of A. M. Davie (unpublished), has zero analytic capacity.

An analogue of the Browder metric density theorem shows that if $\varphi_{\zeta}$ is a distinguished homomorphism, then almost all sequences in $D$ which tend to $\zeta$ must converge to $\varphi_{\zeta}$ in the norm topology of $H^{\infty}(D)^{*}$. It may occur, though, that there is no curve in $D$ terminating at $\zeta$, which converges to $\varphi_{\zeta}$ in the norm topology of $H^{\infty}(D)^{*}$. One geometric condition which guarantees that a sequence $\left\{z_{n}\right\}$ in $D$ tending to $\zeta$ satisfies $\left\|z_{n}-\varphi_{\zeta}\right\| \rightarrow 0$ is that

$$
d\left(z_{n}, \partial D\right) \leqq c\left|z_{n}-\zeta\right|,
$$

where $c>0$ is fixed, and " $d$ " denotes "distance."

Another result from [22] is that if $\left\{z_{n}\right\}$ is a sequence in $D$ which tends to $\zeta \in \partial D$, then either $\left\{z_{n}\right\}$ has a subsequence which is an interpolating sequence, or else $\left\{z_{n}\right\}$ converges in the norm of $H^{\infty}(D)^{*}$ to a distinguished homomorphism $\varphi_{\zeta} \in \mathscr{M}_{\zeta}(D)$. A typical problem relating cluster values and the fibers $\mathscr{M}_{\zeta}(D)$ is as follows. If $\varphi_{\zeta}$ is a distinguished homomorphism, and if $f \in H^{\infty}(D)$ has a limit along a curve in $D$ terminating at $\zeta$, must that limit coincide with $f\left(\varphi_{\zeta}\right)$ ? Garnett (unpublished) has obtained an affirmative answer providing the curve is sufficiently smooth.

Some of the basic results concerning distinguished homomorphisms carry over to a more general setting. One can replace $D$ by a bounded Borel subset $E$ of $C$, and $H^{\infty}(D)$ by a weak-star closed subalgebra of $L^{\infty}\left(\sigma_{E}\right)$ which is $T$-invariant, that is, which is invariant under the " $T_{g}$ "operators used in rational approximation theory.

6. The Silov boundary. A boundary for $H^{\infty}(D)$ is a subset $E$ of $\mathscr{M}(D)$ with the property that every $f \in H^{\infty}(D)$ attains its maximum modulus on $E$. According to Šlov's theorem, there is a minimal closed boundary for $H^{\infty}(D)$, the Silov boundary of $H^{\infty}(D)$, which will be denoted by $\mathrm{w}(D)$. It is easy to show that $Z(\omega(D))$ coincides with the set of essential boundary 
points of $D$. Since the closure of $D$ in $\mathscr{M}(D)$ is a boundary for $H^{\infty}(D), \omega(D)$ is adherent to $D$.

The Šilov boundary $\omega(D)$ for arbitrary $D$ is studied in [17]. There it is shown that if $u$ is a continuous real-valued function on $w(D)$, then there is $f \in H^{\infty}(D)$ such that $|f|=e^{u}$ on $\omega(D)$. Furthermore, $\omega(D)$ is extremely disconnected (the closure of every open subset is open), and the adherence in $\omega(D)$ of any subset of $D$ is a clopen subset of $\omega(D)$. One further fact, which places topological restrictions on $w(D)$, is that there is a (finite) measure whose closed support coincides with $w(D)$. In fact, the following simple lemma plays a crucial role, so we give a complete proof.

Lemma. Suppose $D$ is connected, and $z_{0} \in D$. If $v$ is a finite measure on $\mathrm{w}(D)$ such that $f\left(z_{0}\right)=\int f d v$ for all $f \in H^{\infty}(D)$, then the closed support of $v$ coincides with $\mathrm{w}(D)$.

Proof. Let $E$ be the closed support of $v$. Define

$$
u(\zeta)=\int \frac{Z-z_{0}}{Z-\zeta} d v, \quad \zeta \in D .
$$

Then $u$ is analytic on $D$, and $u\left(z_{0}\right)=1$. If $\zeta \in D$ and $f \in H^{\infty}(D)$, then $\left(Z-z_{0}\right)(f-f(\zeta)) /(Z-\zeta)$ belongs to $H^{\infty}(D)$ and vanishes at $z_{0}$, so that

$$
\int \frac{Z-z_{0}}{Z-\zeta}[f-f(\zeta)] d v=0
$$

Consequently

$$
u(\zeta) f(\zeta)=\int \frac{Z-z_{0}}{Z-\zeta} f d v, \quad f \in H^{\infty}(D) .
$$

If $u(\zeta) \neq 0$, this yields an estimate of the form $|f(\zeta)| \leqq c\|f\|_{E}, f \in H^{\infty}(D)$. Applying this inequality to $f^{n}$, taking $n$th roots, and letting $n \rightarrow \infty$, we obtain $|f(\zeta)| \leqq\|f\|_{E}, f \in H^{\infty}(D)$, whenever $u(\zeta) \neq 0$. The estimate persists for all $\zeta \in D$, so that $\|f\|_{D}=\|f\|_{E}$ for all $f \in H^{\infty}(D)$, and $E$ is a boundary for $H^{\infty}(D)$. Since the Silov boundary is minimal, $E$ coincides with $ш(D)$. Q.E.D.

Now we consider the Dixmier decomposition theorem [12] for extremely disconnected spaces. In view of the preceding lemma, the Dixmier decomposition specializes in the case at hand to the following. There is a unique decomposition

$$
ш(D)=T_{0} \cup T_{1},
$$

where $T_{0}$ and $T_{1}$ are disjoint clopen subsets of $\omega(D)$ with the properties that $T_{0}$ has a dense meager subset, and $T_{1}$ is the closed support of a finite positive normal measure $v$. (By normal, we mean that $v(E)=0$ for every 
set $E$ with empty interior.) The inclusion $C\left(T_{1}\right) \rightarrow L^{\infty}(v)$ is then an isometric isomorphism of the two algebras, so that $T_{1}$ is homeomorphic to the spectrum $\Sigma(v)$ of $L^{\infty}(v)$.

A discussion of the relevant facts concerning the Silov boundary of $H^{\infty}(\Delta)$ is found in [28]. Fatou's theorem allows us to identify $H^{\infty}(\Delta)$ with a subalgebra of $L^{\infty}(d \theta) \cong C(\Sigma(d \theta))$, where $d \theta$ is the arc length measure on $\partial \Delta$. If $u$ is a continuous real-valued function on $\Sigma(d \theta)$, then there is an invertible function $f \in H^{\infty}(\Delta)$ such that $|f|=e^{u}$ on $\Sigma(d \theta)$. It follows that $\Psi(\Delta) \cong \Sigma(d \theta)$. Since the lift of $d \theta$ to $\Sigma(d \theta)$ is a normal measure on $\boldsymbol{w}(\Delta)$, the component $T_{0}$ of (4) is empty, and $w(\Delta)$ coincides with $T_{1}$.

What is the adherence in $\mathrm{w}(\Delta)$ of a subset $S$ of $\Delta$ ? It is precisely that clopen subset of $\omega(\Delta)$ which corresponds to the Borel subset $E$ of $\partial \Delta$ consisting of the nontangential limit points of $S$. In particular, we have the Brown-Shields-Zeller theorem (suggested by G. Piranian; see [4]), that almost all $(d \theta)$ points of $\partial \Delta$ are nontangential limit points of $S$ if and only if $\Psi(\Delta)$ is adherent to $S$, that is, if and only if $\|f\|_{S}=\|f\|_{\Delta}$ for all $f \in H^{\infty}(\Delta)$.

There is an example in [17] of a domain, rather complicated, for which $\Psi(D)$ has a dense meager subset, so that $T_{0}=ш(D)$, and $T_{1}$ is empty.

One open problem along these lines is to identify the normal measure $v$ on $T_{1}$. In particular, can $v$ be taken always to be the restriction to $T_{1}$ of the "harmonic measure" on $\mathscr{M}(D)$ ?

The bidisc algebra $H^{\infty}(\Delta \times \Delta)$ is isometrically isomorphic to a subalgebra of $L^{\infty}(m)$, where $m$ is the area measure on $\partial \Delta \times \partial \Delta$. Let $X$ be the compact space obtained from the spectrum $\Sigma(m)$ of $L^{\infty}(m)$ by identifying points which are identified by $H^{\infty}(\Delta \times \Delta)$. Then $X$ can be regarded as a subset of $\mathscr{M}(\Delta \times \Delta)$, and $X$ contains the Silov boundary $\omega(\Delta \times \Delta)$. The work of R. M. Range and J.-P. Rosay (see [34]) can be sharpened to show that $\omega(\Delta \times \Delta)$ has zero mass for the lift of the measure $m$ to $X$. Otherwise, virtually nothing is known about $\boldsymbol{w}(\Delta \times \Delta)$. One might begin by asking how disconnected $\boldsymbol{m}(\Delta \times \Delta)$ is.

7. Relation to cluster value theory. It turns out that the restriction of $H^{\infty}(D)$ to a fiber $\mathscr{M}_{\zeta}(D)$ is a closed subalgebra of $C\left(\mathscr{M}_{\zeta}(D)\right)$. Its Silov boundary is denoted by $\varpi_{\zeta}(D)$. The principal link between the abstract approach and cluster value theory is the identity

$$
\omega_{\zeta}(D)=\mathscr{M}_{\zeta}(D) \cap \omega(D),
$$

valid whenever $\zeta$ is an essential boundary point of $D$. The difficult inclusion in (5) is proved in [22], and the reverse inclusion, which is valid for domains in $\boldsymbol{C}^{n}$, is noted in [18].

The identity (5), together with the elementary Banach-algebra principle to the effect that "the range on the Šilov boundary includes the boundary 
of the range," yields information on the cluster behavior of analytic functions. One simple conclusion which can be drawn from (5), and which turns out to apply to the Ahlfors function and other extremal functions, is the following. If $f \in H^{\infty}(D)$ is unimodular on $\omega(D)$, and if $\zeta$ is an essential boundary point for $D$, then either

$$
\lim _{D \ni z \rightarrow \zeta}|f(z)|=1, \quad \text { or } \quad \mathrm{Cl}(f, \zeta)=\bar{\Delta} \quad \text { (the closed unit disc). }
$$

Theorems on omitted values also can be linked to the abstract approach. The simplest theorem along these lines asserts that if $f \in H^{\infty}(D)$ and $\zeta \in \partial D$, then $\mathrm{Cl}(f, \zeta) \backslash f\left(\mathrm{w}_{\zeta}(D)\right)$ is an open subset of $C$, and, with the exception of a subset of zero analytic capacity, every value in this set is attained by $f$ on $D$ infinitely often in any neighborhood of $\zeta$.

In [18], the identity (5) is established for polydomains in $\boldsymbol{C}^{n}$. There it is shown how (5) leads to strong versions of the Iversen-Tsuji theorem on cluster values. The version of the theorem obtained for polydomains is described as follows.

Let $\Omega=D_{1} \times \cdots \times D_{n}$ be a polydomain in $C^{n}$, and let $\pi: \Delta^{n} \rightarrow \Omega$ be the universal covering map. A conformal ray in $\Omega$ is the image under $\pi$ of a radial segment $\left\{\left(r e^{i \theta_{1}}, \ldots, r e^{i \theta_{n}}\right): 0 \leqq r<1\right\}$. The family of conformal rays has a natural measure inherited from volume measure on $(\partial \Delta)^{n}$. Almost all conformal rays terminate in $\partial \Omega$, and each $f \in H^{\infty}(\Omega)$ has a limit along almost all conformal rays.

Now let $f \in H^{\infty}(\Omega)$, and let $\zeta_{j}$ be an essential boundary point of $D_{j}$, $1 \leqq j \leqq n$. The theorem asserts that if there is a neighborhood $V$ in $\partial \Omega$ of $\zeta=\left(\zeta_{1}, \ldots, \zeta_{n}\right)$ such that the limit of $f$ along almost all conformal rays terminating in $V$ is bounded in modulus by $M$, then

$$
\lim _{D \ni z \rightarrow \xi}|f(z)| \leqq M .
$$

An open problem is to determine to what extent there is a version of Iversen's theorem valid for arbitrary domains in $\boldsymbol{C}^{n}$.

8. Extremal problems. In the remainder of this report, we assume that $D$ is connected. Fix $z_{0} \in D$, and consider the extremal problem:

$$
\begin{aligned}
& \text { to maximize }\left|f^{\prime}\left(z_{0}\right)\right| \text {, among all } f \in H^{\infty}(D) \text { which } \\
& \text { satisfy }\|f\| \leqq 1 \text {. }
\end{aligned}
$$

The extremal function $G$ for (6), normalized so that $G^{\prime}\left(z_{0}\right)>0$, is unique, and it is called the Ahlfors function for $D$. The Ahlfors function $G$, and the corresponding extremal value $G^{\prime}\left(z_{0}\right)$, play an important role in function theory and in approximation theory.

In the case that $\partial D$ is analytic, L. Ahlfors [1] showed that $G$ extends analytically across $\partial D$, and $G$ has unit modulus on $\partial D$. More generally, if 
$\partial D$ contains a free boundary arc, then $G$ extends continuously to that arc and has unit modulus there [13]. The Ahlfors function tries hard to be unimodular on the boundary of arbitrary domains. The following key theorem to that effect was obtained by S. Fisher [14].

THEOREM. The Ahlfors function for a bounded domain $\boldsymbol{D}$ in $\boldsymbol{C}$ has unit modulus on the Šilov boundary of $H^{\infty}(D)$.

Proof. Evidently $G^{\prime}\left(z_{0}\right)$ is the norm of the linear functional $f \rightarrow f^{\prime}\left(z_{0}\right)$ on $H^{\infty}(D)$. By the Hahn-Banach theorem and the Riesz representation theorem, there is a measure $\eta$ on $w(D)$ such that $\|\eta\|=G^{\prime}\left(z_{0}\right)$ and $f^{\prime}\left(z_{0}\right)=\int f d \eta, f \in H^{\infty}(D)$. If $v=\left(Z-z_{0}\right) \eta$, then $f\left(z_{0}\right)=\int f d v$ for all $f \in H^{\infty}(D)$ so that our lemma ( $\S 6$ ) shows that the closed support of $v$, and hence the closed support of $\eta$, coincides with $w(D)$. The chain of inequalities $G^{\prime}\left(z_{0}\right)=\int G d \eta \leqq \int|G| d|\eta| \leqq\|\eta\|=G^{\prime}\left(z_{0}\right)$ shows that $|G|=1$ a.e. $(d \eta)$, so that $|G|=1$ on $w(D)$. Q.E.D.

Incidentally, the preceding proof also establishes the uniqueness of the Ahlfors function. Indeed, if $G_{0}$ and $G_{1}$ are normalized extremal functions for (4), then any convex combination of $G_{0}$ and $G_{1}$ is extremal. By the theorem, any convex combinations of $G_{0}$ and $G_{1}$ is unimodular on $\mathrm{w}(D)$. It follows that $G_{0}=G_{1}$ on $\Psi(D)$, and hence on $D$.

Combined with the results on cluster value theory described in the preceding section, Fisher's theorem yields information on the Ahlfors function which is already sharper than the information which had been obtained by classical means.

Fisher's theorem can be extended to a wide range of extremal problems. One class of such extremal problems is described as follows.

Let $K$ be a compact subset of $D$. A linear functional on $H^{\infty}(D)$ is $K$-continuous if it is continuous with respect to the topology of uniform convergence on $K$.

Fix $K$-continuous linear functionals $\Lambda, L_{1}, \ldots, L_{m}$ on $H^{\infty}(D)$, and fix complex numbers $a_{1}, \ldots, a_{m}$. Consider the following extremal problem of "Pick-Nevanlinna" type:

to maximize $\operatorname{Re} \Lambda(f)$, among all $f \in H^{\infty}(D)$ which satisfy $\|f\| \leqq 1$ and $L_{j}(f)=a_{j}, 1 \leqq j \leqq m$.

It is shown in [16] that if $\Lambda$ is not a linear combination of $L_{1}, \ldots, L_{m}$, and if there is at least one competing function for (7), then there is a unique extremal function for (7). Moreover, if $K$ does not separate $\partial D$, then the extremal function has unit modulus on the Silov boundary $w(D)$ of $H^{\infty}(D)$, and it extends analytically across any free analytic boundary arcs for $D$.

9. Dual extremal problems. Suppose that $\partial D$ is analytic, that is, that 
$\partial D$ consists of a finite number of disjoint analytic closed Jordan curves. Then there is an isomorphism $L \rightarrow \hat{L}$ between $K$-continuous linear functionals $L$ on $H^{\infty}(D), K$ running over compact subsets of $D$, and germs $\hat{L}$ of analytic functions on $C \backslash D$ which vanish at $\infty$. If $\eta$ is any measure on $K$ which represents $L$, then $\hat{L}$ is given by

$$
\hat{L}(\zeta)=\int \frac{d \eta(z)}{z-\zeta}, \quad \zeta \notin K .
$$

From $\hat{L}$ we can recover $L$ by the formula

$$
L(f)=-\frac{1}{2 \pi i} \int_{\Gamma} \hat{L}(z) f(z) d z, \quad f \in H^{\infty}(D),
$$

where $\Gamma$ is a cycle in $D$ which surrounds the singularities of $\hat{L}$.

Let $d|z|$ denote the arc length measure on $\partial D$, and denote by $H^{1}(d|z|)$ the closure in $L^{1}(d|z|)$ of the space of functions which are analytic on $\bar{D}$. With the extremal problem

$$
\begin{aligned}
& \text { to maximize } \operatorname{Re} L(f) \text {, among } f \in H^{\infty}(D) \text { which satisfy } \\
& \|f\| \leqq 1
\end{aligned}
$$

we associate the dual extremal problem

$$
\text { to minimize } \int|\hat{L}(z)-h(z)| d|z| \text {, among all } h \in H^{1}(d|z|) \text {. }
$$

In other words, the dual extremal problem is to compute the distance from $\hat{L}$ to $H^{1}(d|z|)$, and this is equivalent to the following:

$$
\begin{aligned}
& \text { to minimize } \int|g(z)| d|z| \text {, among all functions } g \text { analytic } \\
& \text { near } \partial D \text { such that } g-\hat{L} \text { extends analytically to } D \text {. }
\end{aligned}
$$

The extremal functions for (10) are called dual extremal functions. They always exist; and the dimension of the convex set of dual extremal functions does not exceed $m-1$, where $m$ is the number of boundary curves of $D$. The dual extremal function is unique just as soon as the extremal function for (8) is not constant. If $L$ is $K$-continuous, where $K$ does not separate $\partial D$, then the dual extremal function $g$ is analytic across $\partial D$, and it satisfies $g(z) G(z) d z \geqq 0$ along $\partial D$, where $G$ is the extremal function for (8). Most of these facts are covered in [20]. For dual extremal problems on Riemann surfaces, see [2] and [36].

The dual extremal function associated with the functional $L(f)=$ $f^{\prime}\left(z_{0}\right)$ is called the Garabedian function. It is analytic on $\bar{D} \backslash\left\{z_{0}\right\}$, and it has a double pole at $z_{0}$. The Garabedian function is introduced in [23], and 
studied in [13] and [26]. Short expository accounts are found in [7] and [25].

The role played by dual extremal functions for arbitrary domains has not been developed. Recently E. Smith [43] settled a problem left open by S. Ya. Havinson [26], proving that if domains $D_{n}$ with analytic boundaries increase to an arbitrary domain $D$, then the Garabedian functions of the $D_{n}$ converge normally. The limit function depends only on $D$ and on the point $z_{0}$, and it is accordingly called the Garabedian function of $D$. In order to study the subadditivity problem for analytic capacity, Smith had been led to investigate the dependence of the Szegö kernel function on certain perturbations of domains with analytic boundaries. The result on the Garabedian function dropped out as a special dividend. There is now the problem of simplifying Smith's proof, and of freeing the result from Hilbert space considerations, in order to extend the theorem to more general extremal problems.

ADDED IN PROOF. A simple proof, which still depends on Hilbert space considerations, has been given by N. Suita, On a metric induced by analytic capacity, Kōdai Math. Sem. Rep. 25 (1973), 215-218.

10. Pointwise bounded approximation. Let $E$ be an arbitrary subset of $\partial D$, and consider the following problem.

When can every $f \in H^{\infty}(D)$ be approximated pointwise on $D$ by a sequence $\left\{f_{n}\right\}$ in $H^{\infty}(D)$ such that each $f_{n}$ extends to be analytic in a neighborhood of $E$, and $\left\|f_{n}\right\| \leqq\|f\|$.

The usual strategy for approaching this problem is to use Vitushkin's constructive techniques to find $\left\{f_{n}\right\}$ as above, except satisfying only $\left\|f_{n}\right\| \leqq c\|f\|$, and then to use a separation argument to reduce the norms. For instance, one can show [11, Theorems 2.2 and 8.3] that if a capacity estimate of the form

$$
\gamma(\Delta(z ; \delta) \backslash D) \leqq c \gamma(\Delta(z ; r \delta) \backslash(D \cup E))
$$

holds for $z \in E$, then (11) is valid. Here $c>0$ and $r \geqq 1$ can depend on $z$, and the estimate need hold only for "most" $z \in E$. For more on this aspect of the algebra $H^{\infty}(D)$, see the references given in [21]. Here we confine ourselves to an indication of one application of these techniques to extremal problems.

An affirmative answer to (11) allows one to approximate extremal functions for $D$ by extremal functions for domains containing $D \cup E$. This approach is used in [20] to prove that if the harmonic measure for $D$ is carried by the union of an at most countable number of boundary 
components of $D$, then the Ahlfors function $G$ for $D$ is inner; that is, the composition $G \circ \pi$ of $G$ with the universal covering map $\pi: \Delta \rightarrow D$ has radial boundary values of unit modulus a.e. $(d \theta)$. Without the hypothesis on the harmonic measure, the Ahlfors function need not be inner, and an example is given in [17] of a domain $D$ with Ahlfors function $G$ satisfying $|G \circ \pi|<1$ a.e. $(d \theta)$ on $\partial \Delta$.

In addition to determining for which $D$ the Ahlfors function $G$ is inner, there is the problem of determining when $G \circ \pi$ is a Blaschke product.

\section{REFERENCES}

1. L. Ahlfors, Bounded analytic functions, Duke Math. J. 14 (1947), 1-11. MR 9, 24.

2. - Open Riemann surfaces and extremal problems on compact subregions, Comment. Math. Helv. 24 (1950), 100-134. MR 12, 90; 13, 1138.

3. M. Behrens, The maximal ideal space of algebras of bounded analytic functions on infinitely connected domains, Trans. Amer. Math. Soc. 161 (1971), 359-380.

4. L. Brown, A. Shields and K. Zeller, On absolutely convergent exponential sums, Trans. Amer. Math. Soc. 96 (1960), 162-183. MR 26 \#332.

5. R. C. Buck, Algebraic properties of classes of analytic functions, Seminars on Analytic Functions, vol. II, Princeton University, 1957, pp. 175-188.

6. L. Carleson, Interpolation by bounded analytic functions and the corona problem, Ann. of Math. (2) 76 (1962), 547-559. MR 25 \#5186.

7. - Selected problems on exceptional sets, Van Nostrand Math. Studies, no. 13, Van Nostrand, Princeton, N.J., 1967. MR 37 \#1576.

8. - The corona theorem, Proc. Fiftieth Scandanavian Congress (Oslo, 1968). Lecture Notes in Math., vol. 118, Springer, Berlin, 1970, pp. 121-132. MR 41 \#8696.

9. I. Cnop, Spectral study of holomorphic functions with bounded growth, Ann. Inst. Fourier (Grenoble) 22 (1972), 293-309.

10. A. M. Davie, Bounded approximation by analytic functions, J. Approximation Theory 6 (1972), 316-319.

11. A. M. Davie, T. Gamelin and J. Garnett, Distance estimates and pointwise bounded density, Trans. Amer. Math. Soc. 175 (1973), 37-68.

12. J. Dixmier, Sur certains espaces considérés par M. H. Stone, Summa Brasil. Math. 2 (1951), 151-182. MR 14, 69.

13. S. Fisher, On Schwarz's lemma and inner functions, Trans. Amer. Math. Soc. 138 (1969), 229-240. MR 39 \#1651.

14. - The moduli of extremal functions, Michigan Math. J. 19 (1972), 179-183.

15. T. Gamelin, Localization of the corona problem, Pacific J. Math. 34 (1970), 73-81. MR 43 \#2482.

16. - Extremal problems in arbitrary domains, Michigan Math. J. 20 (1973), 3-11.

17. —- The Shilov boundary of $H^{\infty}(U)$, Amer. J. Math. (to appear).

18. _- Iversen's theorem and fiber algebras, Pacific J. Math. 46 (1973), 389-414.

19. - Uniform algebras, Prentice-Hall, Englewood Cliffs, N.J., 1969.

20. —_ Lectures on $H^{\infty}(D)$, La Plata Notas de Matemática No. 21 (1972).

21. - Uniform algebras on plane sets, Proc. Sympos. of Approximation Theory, Austin, Texas; Academic Press, New York (to appear).

22. T. Gamelin and J. Garnett, Distinguished homomorphisms and fiber algebras, Amer. J. Math. 42 (1970), 455-474.

23. P. Garabedian, Schwarz's lemma and the Szegö kernel function, Trans. Amer. Math. Soc. 67 (1949), 1-35. MR 11, 340. 
24. J. Garnett, Vitushkin's localization operator, Indiana Univ. Math. J. 20 (1971), 905-907.

25. - Analytic capacity and measure, Lecture Notes in Math., vol. 297, SpringerVerlag, Berlin and New York, 1972.

26. S. Ja. Havinson, Analytic capacity of sets, joint nontriviality of various classes of analytic functions, and the Schwarz lemma in arbitrary domains, Mat. Sb. 54 (96) (1961), 3-50; English transl., Amer. Math. Soc. Transl. (2) 43 (1964), 215-266. MR 25 \# 182.

27. D. Hejhal, Linear extremal problems for analytic functions, Acta Math. 128 (1972), $91-122$.

28. K. Hoffman, Banach spaces of analytic functions, Prentice-Hall Series in Modern Analysis, Prentice-Hall, Englewood Cliffs, N.J., 1962. MR 24 \# A2844.

29. - Bounded analytic functions and Gleason parts, Ann. of Math. (2) 86 (1967), 74-111. MR 35 \#5945.

30. L. Hörmander, Generators for some rings of analytic functions, Bull. Amer. Math. Soc. 73 (1967), 943-949. MR 37 \#1977.

31. S. Kakutani, Rings of analytic functions. Lectures on functions of a complex variable, Univ. of Michigan Press, Ann Arbor, 1955, pp. 71-83. MR 16, 1125.

32. J. Kelleher and B. A. Taylor, An application of the corona theorem to some rings of entire functions, Bull. Amer. Math. Soc. 73 (1967), 246-249. MR 34 \#6096.

33. - Finitely generated ideals in rings of analytic functions, Math. Ann. 193 (1971), $225-237$.

34. R. M. Range, A small boundary for $H^{\infty}$ on the polydisc, Proc. Amer. Math. Soc. 32 (1972), 253-255. MR 44 \# 7300.

35. W. W. Rogosinski and H. S. Shapiro, On certain extremum problems for analytic functions, Acta Math. 90 (1953), 287-318. MR 15, 516.

36. H. Royden, The boundary values of analytic and harmonic functions, Math. Z. 78 (1962), 1-24. MR 25 \#2190.

37. L. Rubel, Lectures on vector spaces of analytic functions, Symposia on theoretic physics and mathematics (Madras, India), Plenum Press, New York, 1969, pp. 191-269.

38. - B Bounded convergence of analytic functions, Bull. Amer. Math. Soc. 77 (1971), 13-24. MR 42 \# 5025.

39. L. Rubel and J. Ryff, The bounded weak-star topology and the bounded analytic functions, J. Functional Analysis 5 (1970), 167-183. MR 40 \#7788.

40. L. Rubel and A. Shields, The space of bounded analytic functions on a region, Ann. Inst. Fourier (Grenoble) 16 (1966), fasc. 1, 235-277. MR 33 \#6440.

41. W. Rudin, Essential boundary points, Bull. Amer. Math. Soc. 70 (1964), 321-324. MR 28 \#3167.

42. D. Sarason, Algebras of functions on the unit circle, Bull. Amer. Math. Soc. 79 (1973). 286-299.

43. E. Smith, The Garabedian function of an arbitrary compact set, Pacific J. Math. (to appear).

44. E. L. Stout, Two theorems concerning functions holomorphic on multiply connected domains, Bull. Amer. Math. Soc. 69 (1963), 527-530. MR 27 \#275.

45. M. Tsuji, Potential theory in modern function theory, Maruzen, Tokyo, 1959.

46. M. Weiss, Cluster sets of bounded analytic functions from a Banach algebraic viewpoint, Ann. Acad. Sci. Fenn. Ser. AI No. 367 (1965), 14 pp. MR 35 \# 379.

47. L. Zalcman, Bounded analytic functions on domains of infinite connectivity, Trans. Amer. Math. Soc. 144 (1969), 241-269. MR 40 \#5884.

Department of Mathematics, University of California, los Angeles, California 90024 